\title{
Endovascular versus open repair of ruptured descending thoracic aortic aneurysms: A nationwide risk-adjusted study of 923 patients
}

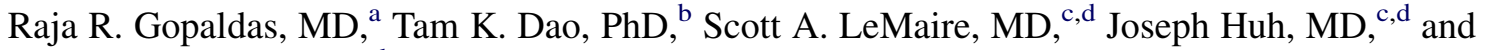 \\ Joseph S. Coselli, MD ${ }^{\mathrm{c}, \mathrm{d}}$
}

\begin{abstract}
Objective: Recent studies support the use of endovascular treatment for ruptured abdominal aortic aneurysms, but few studies have examined the use of thoracic endovascular aortic repair (TEVAR) for ruptured descending thoracic aortic aneurysm. We evaluated nationwide data regarding short-term outcomes of TEVAR and open aortic repair (OAR) for ruptured descending thoracic aortic aneurysm.
\end{abstract}

\begin{abstract}
Methods: From US Nationwide Inpatient Sample data, we identified 923 patients who underwent ruptured descending thoracic aortic aneurysm repair in 2006-2008 and who had no concomitant aortic disorders. Of these patients, 364 (39.4\%) underwent TEVAR and 559 (60.6\%) underwent OAR. Multivariable regression was used to assess the effect of TEVAR versus OAR after adjusting for potential confounding factors. Outcomes assessed were in-hospital mortality, complications, failure to rescue (defined as the mortality among patients in whom a complication develops), and disposition. Backward stepwise logistic regression was used to identify independent predictors of outcomes for each approach.
\end{abstract}

Results: Patients undergoing TEVAR were older $(72 \pm 12$ years vs $65 \pm 15$ years; $P<.001)$ and had a higher Deyo comorbidity index $(4.19 \pm 1.79$ vs $3.14 \pm 2.05 ; P<.001)$ than patients undergoing OAR. Unadjusted mortality was $23.4 \%$ (85/364) for TEVAR and $28.6 \%(160 / 559)$ for OAR. After risk adjustment, the odds of mortality, complications, and failure to rescue were similar for TEVAR and OAR $(P>.1$ for all), but patients undergoing TEVAR had a greater chance of routine discharge (odds ratio $[\mathrm{OR}]=3.3 ; P<.001)$. An interaction was identified that linked hospital size and operative approach with risk of complications $(P<.001)$. In smaller hospitals, TEVAR was associated with lower complication rates than OAR $(\mathrm{OR}=0.21 ; P<.05)$. Regression analysis revealed that smaller hospital size predicted significantly higher rates of mortality $(\mathrm{OR}=2.4 ; P<.05)$, complications $(\mathrm{OR}=4.0 ; P<.005)$, and failure to rescue $(\mathrm{OR}=51.12 ; P<.001)$ in those undergoing OAR but not in those undergoing TEVAR. Preexisting renal disorders substantially increased mortality risk $(\mathrm{OR}=10.81 ; P<.001)$ and failure to rescue $(\mathrm{OR}=309.54 ; P<.001)$ in patients undergoing TEVAR.

Conclusions: Nationwide data for ruptured descending thoracic aortic aneurysm reveal equivalent mortality, complication rates, and failure to rescue for TEVAR and OAR but more frequent routine discharge with TEVAR. Unlike OAR outcomes, TEVAR outcomes were not poorer in smaller hospitals, where TEVAR produced fewer complications than OAR. Therefore, TEVAR may be an ideal alternative to OAR for ruptured descending thoracic aortic aneurysm, particularly in small hospitals where expertise in OAR may be lacking and immediate transfer to a higher echelon of care may not be feasible. (J Thorac Cardiovasc Surg 2011;142:1010-8)

Thoracic endovascular aortic repair (TEVAR) has gained popularity in the United States since the Food and Drug Administration approved the first TEVAR endoprosthesis in 2005 . $^{1}$ Today, the technique is commonly used in patients with suitable anatomy. Recent studies have shown the feasibility and superior outcomes of TEVAR in single centers and nationwide. ${ }^{2,3}$ A nationwide study recently showed not only

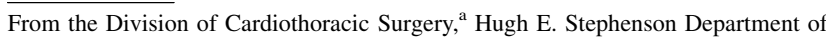
Surgery, University of Missouri-Columbia School of Medicine, Columbia, Mo; Department of Educational Psychology, ${ }^{\mathrm{b}}$ University of Houston, Houston, Tex; Division of Cardiothoracic Surgery, ${ }^{\mathrm{c}}$ Michael E. DeBakey Department of Surgery, Baylor College of Medicine, Houston, Tex; and The Texas Heart Institute at St Luke's Episcopal Hospital, ${ }^{\mathrm{d}}$ Houston, Tex.

Disclosures: Authors have nothing to disclose with regard to commercial support. Read at the 91st Annual Meeting of The American Association for Thoracic Surgery, Philadelphia, Pennsylvania, May 7-11, 2011. wider adoption of TEVAR but also a reduction in riskadjusted mortality between 2005 and 2007. ${ }^{4}$ The use of TEVAR has expanded to several off-label indications, such as dissections. $^{5-8}$ Yet, the use of TEVAR in patients with ruptured thoracic aneurysms has not been studied in detail, and this application of TEVAR has not become nearly as popular as the use of TEVAR for elective thoracic

Received for publication May 9, 2011; revisions received July 21, 2011; accepted for publication Aug 10, 2011; available ahead of print Sept 12, 2011.

Address for reprints: Raja R. Gopaldas, MD, Assistant Professor of Cardiothoracic Surgery, Division of Cardiothoracic Surgery, Suite MA 312, University of Missouri-Columbia School of Medicine, Columbia, MO 65212 (E-mail: gopaldasr@health.missouri.edu). 0022-5223/\$36.00

Copyright (C) 2011 by The American Association for Thoracic Surgery doi:10.1016/j.jtcvs.2011.08.014 


\section{Abbreviations and Acronyms \\ FTR = failure to rescue (defined as death after a complication) \\ ICD-9-CM = International Classification of Diseases, Ninth Revision, Clinical Modification \\ NIS $=$ Nationwide Inpatient Sample \\ OAR $=$ open aortic repair \\ OR $=$ odds ratio \\ rDTAA $=$ ruptured descending thoracic aortic aneurysm \\ TEVAR $=$ thoracic endovascular aortic repair}

aneurysm repair. Some single-center studies recently evaluated the use of TEVAR for ruptured aneurysms, ${ }^{9,10}$ and a population-based study showed that endovascular techniques produced better results than open surgical repair for ruptured abdominal aortic aneurysm. ${ }^{11}$

Compared with traditional open surgery, TEVAR has been associated with a lower risk of a composite of death, stroke, and paraplegia. ${ }^{12}$ Studies have found that for a ruptured descending thoracic aortic aneurysm (rDTAA), TEVAR produces results equivalent to those of open repair whenever TEVAR is feasible. ${ }^{12,13}$ However, these studies were restricted to a few centers of excellence and had small patient cohorts. The relative utility of TEVAR in elective cases is a topic of contention; population-based studies suggest that endovascular approaches produce superior outcomes, whereas results from single centers of excellence continue to favor the traditional open approach. ${ }^{2,14,15}$ Thus, little is known about TEVAR's nationwide use and outcomes as a treatment for rDTAA. Therefore, we evaluated the in-hospital outcomes of TEVAR and open procedures performed for rDTAA in the United States during the initial 3-year period after Food and Drug Administration approval. We also attempted to identify hospital characteristics that affect outcomes.

\section{MATERIALS AND METHODS}

\section{Data Source}

We obtained patient data from the US Nationwide Inpatient Sample (NIS), a database of hospital inpatient stays that is maintained by the Agency for Healthcare Research and Quality as a part of the Healthcare Cost and Utilization Project. ${ }^{16}$ Data from calendar years 2006 to 2008 were obtained. The NIS is the largest all-payer inpatient care database, representing $90 \%$ of all hospital discharges from facilities in the United States except government hospitals and Veterans Affairs medical centers. The NIS has numerous internal quality assurance procedures for ensuring the consistency and validity of data (available at: www.hcup-us.ahrq.gov/db/ quality.jsp). ${ }^{17}$ The NIS contains data on approximately 8 million hospital stays annually from more than 1000 hospitals, although the exact number varies slightly on an annual basis. Only data from participating states were included; 38, 40, and 42 states participated in the NIS for the years 2006,
2007, and 2008, respectively. Weights based on sampling probabilities for each stratum were used in the analysis to ensure that the hospitals studied were representative of all US hospitals.

The variables available in the NIS database include patient and hospital demographics, hospital ownership, payer information, treating and concomitant diagnoses, inpatient procedures, in-hospital mortality, and hospital length of stay (ie, the date of admission to the date of discharge). The NIS database also captures up to 15 International Classification of Diseases, Ninth Revision, Clinical Modification (ICD-9-CM) diagnostic and procedure codes per admission. ${ }^{18}$ The study was approved by the institutional review board of the University of Missouri-Columbia, which deemed the study exempt from the informed consent requirement because the data were nonidentifiable. The reported data conformed to the Healthcare Cost and Utilization Project data-use agreement for the NIS. Additional information about the NIS is available from the Agency for Healthcare Research and Quality (available at: www.hcup-us.ahrq.gov/nisoverview.jsp).

\section{Patient Selection}

From the weighted NIS data for the calendar years 2006-2008, we used the ICD-9-CM diagnosis and procedure codes (Appendix 1$)^{18}$ to identify all patients aged 18 years or older who had a diagnosis of rDTAA and had undergone open aortic repair (OAR) or TEVAR in an urgent or emergency admission. Patients with vasculitis, any connective tissue disorder, gonadal dysgenesis, aortic dissection, or concomitant aneurysms in other aortic segments were excluded. Patients who had undergone both OAR and TEVAR were also excluded because we could not determine from the available data whether these were intraoperative conversions or planned staged procedures. The selection methods were similar to those used in previous population-based TEVAR studies and excluded all patients who underwent total cardiopulmonary bypass. ${ }^{2,19}$

The Deyo index was used to compare the 2 groups in terms of preoperative morbidity. ${ }^{20}$ The Deyo index - a weighted comorbidity index modified from the Charlson comorbidity index-assesses 17 prespecified comorbidities and is specifically designed to be used with administrative databases, using more than 600 ICD-9-CM diagnosis codes to query specific comorbid diagnoses. ${ }^{21}$ In previous surgical studies, we and other investigators have demonstrated the feasibility of querying the NIS database for cardiac, aortic, and vascular procedures. ${ }^{2,22-25}$ Up to 15 ICD-9-CM diagnosis codes were recorded per patient. These codes were then used to produce a summary Deyo index score for each patient. The Deyo index and its individual components were used for risk adjustment in the statistical model.

\section{Study End Points}

The primary outcomes of interest for the present study were in-hospital mortality (defined as death during the index hospitalization), complications, failure to rescue (FTR; defined as mortality among patients in whom a complication develops), and discharge disposition. FTR typically reflects hospital performance and quality of care; hospitals that provide the highest quality of care are more capable of rescuing patients in whom a complication develops and therefore tend to have lower FTR rates. Discharge disposition was classified as routine or nonroutine. A routine discharge was defined as discharge to home without any sort of home health care, including home intravenous medication therapy. Nonroutine discharges included discharge to a skilled nursing or intermediate care facility or to home with home health care, as well as departure from the hospital against medical advice.

All-cause in-hospital morbidity was recorded according to the ICD-9CM diagnosis codes. The following morbidity categories were assessed: intraoperative/procedure-related complications, postoperative infections, neurologic complications, renal complications, respiratory complications, deep vein thrombosis, and pulmonary embolism. Intraoperative and procedure-related complications were defined as complications that occurred during a procedure or that were directly related to technical aspects of the procedure (Appendix 2). 


\section{Statistical Analysis}

The Statistical Package for Social Sciences, version 18.0 (IBM SPSS, Chicago, Ill), complex module was used for all statistical analyses. The Student $t$ test and chi-square statistics were computed to examine intergroup differences in age, race, sex, patient income (by quartile, as encoded in the NIS databases), Deyo index score, mortality, complications, hospital length of stay, and discharge status. After univariate analysis, multivariable analysis with hierarchic regression methods was performed.

Hierarchic logistic regression was used to examine the risk-adjusted association of procedure type (OAR vs TEVAR) with in-hospital complications, mortality, FTR, and discharge disposition. From the results of this overall regression analysis, an interaction analysis model was created to identify the relationship between the use of TEVAR and the hospital size. The NIS stratifies hospital bed size into 3 categories according to geographic region, hospital teaching status, and rural versus urban location. The cutoff values used to define hospital size vary accordingly and can be found on the Agency for Healthcare Research and Quality website (http://www.ahrq.gov/). ${ }^{16}$ For ease of statistical analysis, the small and medium bed size strata were combined into 1 group that was referred to as the "smaller bed size hospital" group. A subgroup analysis was then performed to examine the impact of hospital bed size on each treatment group (OAR and TEVAR). A backward stepwise regression model with individual components of the Deyo score was used for this subgroup analysis. The iteration for the regression equation was run in a stepwise fashion according to the change in the likelihood ratio. The model thus eliminated in a stepwise fashion variables that did not contribute to the outcome until the least change in likelihood ratio was achieved. Stringent variable inclusion and exclusion thresholds ( 0.01 for "in" and 0.1 for "out") were used to create a conservative model. This ensured that the model would summarize the covariates in a conservative manner that would be unlikely to produce significant results by chance.

\section{RESULTS \\ Demographics and Baseline Characteristics}

Of the 923 patients who underwent urgent intervention for rDTAA, $364(39.4 \%)$ underwent TEVAR and 559 $(60.6 \%)$ underwent OAR. Several differences were noted between the TEVAR and OAR groups (Table 1): Patients in the TEVAR group were significantly older by 7 years, and a higher proportion of them were women, white, and receiving Medicare. The TEVAR group had significantly higher Deyo comorbidity scores than the OAR group. There was a steady increase in the proportion of patients treated with TEVAR during the study period, increasing from $31 \%$ in 2006 to $47 \%$ in 2008 .

\section{Unadjusted Outcomes}

Although there was a trend toward higher mortality in the OAR group than in the TEVAR group, this difference was not statistically significant (Table 2 ). There were no significant differences in the overall complication rate or FTR between the 2 groups, although patients who underwent TEVAR had shorter hospital stays and a higher incidence of routine home discharge than patients who underwent OAR.

\section{Logistic Regression: Risk-Adjusted Outcomes}

Overall, female sex was a significant independent predictor of overall complications (odds ratio $[\mathrm{OR}]=1.6$ ),
TABLE 1. Baseline characteristics

\begin{tabular}{|c|c|c|c|}
\hline & $\begin{array}{l}\text { Open repair } \\
(\mathbf{n}=\mathbf{5 5 9})\end{array}$ & $\begin{array}{c}\text { TEVAR } \\
(\mathbf{n}=\mathbf{3 6 4})\end{array}$ & $P$ value \\
\hline Age & $64.8 \pm 15.2$ & $72.1 \pm 11.6$ & $<.001$ \\
\hline Female sex & $237(42.4 \%)$ & $181(49.7 \%)$ & .03 \\
\hline Caucasian race & $269(71.2 \%)$ & $225(78.9 \%)$ & .02 \\
\hline $\begin{array}{l}\text { Primary expected } \\
\text { payer-Medicare }\end{array}$ & $325(58.6 \%)$ & $268(73.6 \%)$ & $<.001$ \\
\hline Household income & & & .13 \\
\hline Lowest quartile & $127(23.3 \%)$ & $69(19.2 \%)$ & \\
\hline Second quartile & $151(27.7 \%)$ & $98(27.3 \%)$ & \\
\hline Third quartile & $132(24.2 \%)$ & $111(30.9 \%)$ & \\
\hline Highest quartile & $135(24.8 \%)$ & $81(22.6 \%)$ & \\
\hline Calendar year* & & & $<.001$ \\
\hline 2006 & $248(69.3 \%)$ & $110(30.7 \%)$ & \\
\hline 2007 & $132(57.6 \%)$ & $97(42.4 \%)$ & \\
\hline 2008 & $180(53.3 \%)$ & $158(46.7 \%)$ & \\
\hline Large hospital bed size & $436(77.9 \%)$ & $325(89.0 \%)$ & $<.001$ \\
\hline $\begin{array}{l}\text { Chronic peripheral vascular } \\
\text { disease }\end{array}$ & $20(3.6 \%)$ & $38(10.4 \%)$ & $<.001$ \\
\hline Prior myocardial infarction & $\leq 10(\leq 1.8 \%)$ & $\leq 10(\leq 2.7 \%)$ & $<.01$ \\
\hline Congestive heart failure & $94(16.8 \%)$ & $67(18.4 \%)$ & .53 \\
\hline Cerebrovascular disease & $45(8.1 \%)$ & $35(9.6 \%)$ & .41 \\
\hline Dementia & $\leq 10(\leq 1.8 \%)$ & $\leq 10(\leq 2.7 \%)$ & $<.01$ \\
\hline $\begin{array}{l}\text { Chronic obstructive } \\
\text { pulmonary disease }\end{array}$ & $100(17.9 \%)$ & $105(28.8 \%)$ & $<.001$ \\
\hline Rheumatoid disease & $16(2.9 \%)$ & $\leq 10(\leq 2.7 \%)$ & .14 \\
\hline Peptic ulcer disease & $\leq 10(\leq 1.8 \%)$ & $\leq 10(\leq 2.7 \%)$ & .01 \\
\hline Chronic liver disease & $\leq 10(\leq 1.8 \%)$ & $\leq 10(\leq 2.7 \%)$ & $<.01$ \\
\hline Diabetes mellitus & $39(7.0 \%)$ & $47(12.9 \%)$ & $<.01$ \\
\hline Prior hemiplegia or paraplegia & $36(6.4 \%)$ & $20(5.5 \%)$ & .56 \\
\hline Chronic renal disease & $60(10.7 \%)$ & $49(13.5 \%)$ & .21 \\
\hline Malignancy & $15(2.7 \%)$ & $\leq 10(\leq 2.7 \%)$ & .09 \\
\hline Deyo score & $3.14 \pm 2.04$ & $4.19 \pm 1.79$ & $<.001$ \\
\hline
\end{tabular}

*Percentages are reported within each calendar year.

mortality $(\mathrm{OR}=2.9)$, and FTR $(\mathrm{OR}=5.0)(P<.01$ for all) (Table 3). The use of TEVAR had no impact on mortality, FTR, or complication rates but was associated with a 3 times greater likelihood of routine home discharge $(P<.001)$. In contrast, smaller hospital bed size was associated with higher FTR and complication rates $(P<.05$ and $<.001$, respectively), but not with significantly higher mortality $(P=.06)$, although there was a trend in that direction. To characterize this discrepancy, an interaction analysis was performed.

\section{Interaction Analysis: Type of Procedure and Hospital Bed Size}

An interaction term between hospital size and procedure type was computed to determine whether these variables had a synergistic beneficial or negative effect (Table 4). The interaction factor was not significantly associated with mortality or FTR but was associated with $77 \%$ less complications $(\mathrm{OR}=0.23 ; P=.004)$. The final $\mathrm{OR}$ for 
TABLE 2. Unadjusted outcomes

\begin{tabular}{|c|c|c|c|c|c|}
\hline & Open repair $(n=559)$ & $\operatorname{TEVAR}(n=364)$ & Total $(\mathbf{N}=\mathbf{9 2 3})$ & Chi-square/t & $P$ value \\
\hline In-hospital mortality & $160(28.9 \%)$ & $85(23.4 \%)$ & $245(26.7 \%)$ & 3.432 & .064 \\
\hline Hemopericardium & $41(7.3 \%)$ & $\leq 10(\leq 2.7 \%)$ & $41(4.4 \%)$ & 27.939 & $<.001$ \\
\hline Open cardiac massage & $15(2.7 \%)$ & $\leq 10(\leq 2.7 \%)$ & $15(1.6 \%)$ & 9.929 & .002 \\
\hline $\begin{array}{l}\text { Procedure-related } \\
\text { complications }\end{array}$ & $120(21.5 \%)$ & $54(14.8 \%)$ & $174(18.8 \%)$ & 6.432 & .011 \\
\hline Deep venous thrombosis & $\leq 10(\leq 1.8 \%)$ & $\leq 10(\leq 2.7 \%)$ & $\leq 10(\leq 1.1 \%)$ & 7.720 & .005 \\
\hline Infections & $70(12.5 \%)$ & $62(17.0 \%)$ & $132(14.3 \%)$ & 3.660 & .056 \\
\hline Mediastinitis & $\leq 10(\leq 1.8 \%)$ & $\leq 10(\leq 2.7 \%)$ & $\leq 10(\leq 1.1 \%)$ & 6.571 & .010 \\
\hline Neurologic complications & $42(7.5 \%)$ & $25(6.9 \%)$ & $67(7.3 \%)$ & .131 & .717 \\
\hline Pneumothorax & $15(2.7 \%)$ & $\leq 10(\leq 2.7 \%)$ & $20(2.2 \%)$ & 1.784 & .182 \\
\hline Respiratory complications & $241(43.0 \%)$ & $229(62.9 \%)$ & $470(50.9 \%)$ & 34.872 & $<.001$ \\
\hline Renal complications & $141(25.2 \%)$ & $81(22.3 \%)$ & $222(24.1 \%)$ & 1.065 & .302 \\
\hline Disposition & $139(25.1 \%)$ & $120(33.0 \%)$ & $259(28.2 \%)$ & 6.729 & .009 \\
\hline Overall complications & $380(68.0 \%)$ & $242(66.5 \%)$ & $622(67.4 \%)$ & .224 & .636 \\
\hline FTR & $102(18.4 \%)$ & $62(17.0 \%)$ & $164(17.9 \%)$ & .285 & .594 \\
\hline Length of stay & $17.36(28.0 \%)$ & $14.47(15.5 \%)$ & $15.6(21.4 \%)$ & 2.008 & .045 \\
\hline
\end{tabular}

complications with TEVAR in small hospitals was calculated as an exponent of the combined OR: Exp $(-1.442-$ $0.099)=0.214$. The calculated $95 \%$ confidence interval

TABLE 3. Overall regression analysis summary

\begin{tabular}{|c|c|c|c|c|c|}
\hline \multirow[b]{2}{*}{ Outcome } & \multirow[b]{2}{*}{ Predictor } & \multirow[b]{2}{*}{ OR } & \multicolumn{2}{|c|}{$95 \%$ CI } & \multirow[b]{2}{*}{$P$ value } \\
\hline & & & Lower & Upper & \\
\hline \multirow[t]{6}{*}{ Mortality } & Female & 2.94 & 1.99 & 4.36 & $<.001$ \\
\hline & Income quartile & .62 & .42 & .91 & .015 \\
\hline & $\begin{array}{l}\text { Smaller (vs larger) } \\
\text { hospital }\end{array}$ & 1.58 & .97 & 2.55 & .064 \\
\hline & TEVAR (vs OAR) & 1.04 & .71 & 1.52 & 847 \\
\hline & Deyo index & 96 & .87 & 1.06 & 435 \\
\hline & Caucasian race & 1.92 & 1.21 & 3.04 & .006 \\
\hline \multirow[t]{6}{*}{ Complications } & Female & 1.63 & 1.16 & 2.29 & .005 \\
\hline & Income quartile & 1.30 & .92 & 1.83 & .138 \\
\hline & $\begin{array}{l}\text { Smaller (vs larger) } \\
\text { hospital }\end{array}$ & 1.74 & 1.09 & 2.77 & .021 \\
\hline & TEVAR (vs OAR) & .75 & .53 & 1.06 & .104 \\
\hline & Deyo index & 1.10 & 1.01 & 1.20 & .030 \\
\hline & Caucasian race & .48 & .31 & .71 & $<.001$ \\
\hline \multirow[t]{6}{*}{ FTR } & Female & 5.05 & 2.96 & 8.61 & $<.001$ \\
\hline & Income quartile & .89 & .55 & 1.42 & .611 \\
\hline & $\begin{array}{l}\text { Smaller (vs larger) } \\
\text { hospital }\end{array}$ & 3.44 & 1.97 & 6.04 & $<.001$ \\
\hline & TEVAR (vs OAR) & .99 & .62 & 1.57 & .949 \\
\hline & Deyo index & 1.32 & 1.18 & 1.48 & $<.001$ \\
\hline & Caucasian race & 1.33 & .76 & 2.32 & .319 \\
\hline \multirow[t]{6}{*}{ Disposition } & Female & .47 & .32 & .68 & $<.001$ \\
\hline & Income quartile & 1.37 & .94 & 2.00 & .101 \\
\hline & $\begin{array}{l}\text { Smaller (vs larger) } \\
\text { hospital }\end{array}$ & .52 & .31 & .89 & .017 \\
\hline & TEVAR (vs OAR) & 3.31 & 2.22 & 4.94 & $<.001$ \\
\hline & Deyo index & .76 & .68 & .85 & $<.001$ \\
\hline & Caucasian race & .66 & .44 & 1.00 & .050 \\
\hline
\end{tabular}

$C I$, Confidence interval; TEVAR, thoracic endovascular aortic repair; $O A R$, open aortic repair. did not include 1 , so the OR was considered statistically significant. An independent subgroup analysis was therefore conducted to examine the impact of hospital size on the OAR and TEVAR subgroups separately.

\section{Postinteraction Subgroup Analysis}

The final iteration of the backward stepwise regression model (Table 5) for the TEVAR subgroup revealed that hospital bed size was not predictive of any of the outcomes (mortality, complications, or FTR). In contrast, coexisting renal disease independently predicted mortality and FTR in the TEVAR group. The same statistical analysis performed on the OAR subgroup associated smaller hospital bed size with greater odds of death, complications, and FTR.

\section{DISCUSSION}

This is the largest population-based study to evaluate the outcomes of TEVAR for rDTAA in the United States. It is interesting to note that this rather new technology has been used for an emergency intervention in a substantial number of patients. The TEVAR group differed from the OAR group in that patients in the TEVAR group were older (and, consequently, more likely to use Medicare) and had more preoperative comorbidities. Nonetheless, most of the overall unadjusted outcomes were not significantly different between groups, and the TEVAR group had a higher proportion of routine home discharges than the OAR group.

In earlier multicenter and nationwide studies, TEVAR had been shown to have superior outcomes to OAR even in much older patients. ${ }^{1,2}$ However, its use in patients with rDTAA had not been evaluated on a nationwide basis. Our analysis revealed that, after risk adjustment, TEVAR has equivalent outcomes to OAR but is associated with a greater chance of routine home disposition. In the current era of increasing health care costs, tightening budgets, and an 
TABLE 4. Interaction analysis summary: Hospital bed size and procedure type

\begin{tabular}{|c|c|c|c|c|c|}
\hline \multirow[b]{2}{*}{ Outcome } & \multirow[b]{2}{*}{ Predictor } & \multirow[b]{2}{*}{ OR } & \multicolumn{2}{|c|}{$95 \% \mathrm{CI}$} & \multirow[b]{2}{*}{$P$ value } \\
\hline & & & Lower & Upper & \\
\hline \multirow[t]{7}{*}{ Mortality } & Female sex & 2.94 & 1.99 & 4.35 & $<.001$ \\
\hline & Income quartile & .63 & .42 & .92 & .018 \\
\hline & $\begin{array}{l}\text { Smaller (vs larger) } \\
\text { hospital }\end{array}$ & 1.47 & .79 & 2.75 & .225 \\
\hline & Interaction factor* & 1.19 & .44 & 3.19 & .733 \\
\hline & TEVAR (vs OAR) & 1.01 & .67 & 1.53 & .963 \\
\hline & Deyo index & .96 & .87 & 1.06 & .409 \\
\hline & Caucasian race & 1.92 & 1.21 & 3.04 & .005 \\
\hline \multirow[t]{7}{*}{ Complications } & Female sex & 1.64 & 1.17 & 2.31 & .005 \\
\hline & Income quartile & 1.22 & .86 & 1.73 & .260 \\
\hline & $\begin{array}{l}\text { Smaller (vs larger) } \\
\text { hospital }\end{array}$ & 3.17 & 1.65 & 6.11 & .001 \\
\hline & Interaction factor* & .24 & .09 & .62 & .004 \\
\hline & TEVAR (vs OAR) & .90 & .63 & 1.31 & .601 \\
\hline & Deyo index & 1.12 & 1.03 & 1.23 & .011 \\
\hline & Caucasian race & .46 & .30 & .69 & $<.001$ \\
\hline \multirow[t]{7}{*}{ FTR } & Female sex & 5.10 & 2.98 & 8.72 & $<.001$ \\
\hline & Income quartile & .87 & .54 & 1.40 & .569 \\
\hline & $\begin{array}{l}\text { Smaller (vs larger) } \\
\text { hospital }\end{array}$ & 3.87 & 1.80 & 8.33 & .001 \\
\hline & Interaction factor* & .78 & .26 & 2.39 & .664 \\
\hline & TEVAR (vs OAR) & 1.04 & .62 & 1.75 & .885 \\
\hline & Deyo index & 1.33 & 1.18 & 1.49 & $<.001$ \\
\hline & Caucasian race & 1.32 & .75 & 2.30 & .335 \\
\hline
\end{tabular}

$C I$, Confidence interval; TEVAR, thoracic endovascular aortic repair; $O A R$, open aortic repair; FTR, failure to rescue. *Refers to the interaction between smaller hospital bed size and the use of TEVAR.

aging baby-boomer population, the higher rate of routine disposition constitutes an important advantage of TEVAR, as does the shorter hospital stay associated with this intervention.

Smaller hospital bed size was associated with greater odds of developing a complication after surgical intervention for rDTAA, yet the TEVAR technique by itself did not increase complication rates. Rather, when TEVAR was performed in smaller hospitals, the overall complication rate was lower. This synergistic effect was not seen with FTR or mortality, leading us to conclude that smaller hospitals are still less capable than larger hospitals of handling the complications of TEVAR or OAR. However, it seems probable that TEVAR less often results in complications that would be difficult for small hospitals to handle. More broadly, the interaction between hospital bed size and the use of new technology such as TEVAR may be advantageous for smaller hospitals. This is because the cardiac surgical, operating room, and intensive care unit personnel at smaller hospitals may not have the skill sets and experience of their counterparts at larger hospitals, resulting in poorer outcomes of OAR at smaller hospitals. Conversely, small hospitals that perform TEVAR generally have a cardiac catheterization laboratory or endovascular suite with appropriate ancillary medical personnel who can provide better support services than the personnel involved in OAR procedures at the same hospital. Thus, it is probably less difficult for a small hospital to handle straightforward TEVAR than OAR procedures, so that the procedural aspects of TEVAR are performed better than those of OAR in smaller hospitals.

The discrepancy in outcomes between TEVAR and OAR performed at smaller hospitals was not seen in larger hospitals, which seemed to be equally competent in both techniques. Thus, TEVAR performed at smaller hospitals, TEVAR performed at larger hospitals, and OAR performed at larger hospitals all seem to produce similar outcomes, whereas OAR performed at smaller hospitals produces poorer outcomes. In light of these findings, it may be best to avoid OAR at a smaller hospital, even if safe transfer to a larger facility is not possible. If the patient's aortic anatomy makes TEVAR feasible, and if personnel with endovascular skills are available, using TEVAR in a smaller hospital may be considered. Nonetheless, if transfer to a larger facility can be accomplished safely, then transfer is the most desirable course of action because of the possibility that conversion from TEVAR to OAR will be necessary. These considerations, along with the higher FTR and mortality associated with TEVAR in patients with preexisting renal disorders, complicate the workup of patients

TABLE 5. Backward stepwise regression: Final iterations of subgroup analysis

\begin{tabular}{|c|c|c|c|c|c|c|}
\hline \multirow[b]{2}{*}{ Predictor } & \multirow[b]{2}{*}{ Outcome } & \multirow[b]{2}{*}{ Group } & \multirow[b]{2}{*}{ OR } & \multicolumn{2}{|c|}{$\mathbf{9 5} \%$ CI } & \multirow[b]{2}{*}{$P$ value } \\
\hline & & & & Lower & Upper & \\
\hline \multirow[t]{3}{*}{ Smaller (vs larger) hospital } & Mortality & OAR & 2.39 & 1.13 & 5.09 & .023 \\
\hline & Complications & OAR & 3.96 & 1.78 & 8.79 & .001 \\
\hline & FTR & OAR & 51.11 & 9.73 & 268.35 & $<.001$ \\
\hline \multirow[t]{3}{*}{ Smaller (vs larger) hospital } & Mortality & TEVAR & 1.00 & .30 & 3.30 & $.997^{*}$ \\
\hline & Complications & TEVAR & .58 & .21 & 1.56 & $.283^{*}$ \\
\hline & FTR & TEVAR & 1.05 & .21 & 5.16 & $.951^{*}$ \\
\hline \multirow[t]{2}{*}{ Renal comorbidity } & Mortality & TEVAR & 10.81 & 3.54 & 32.99 & $<.001$ \\
\hline & FTR & TEVAR & 309.54 & 47.97 & 1997.15 & $<.001$ \\
\hline
\end{tabular}

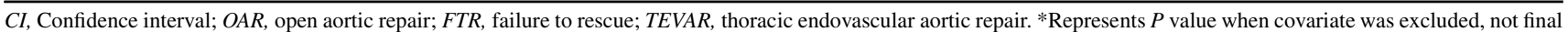
iteration. 
by emergency department physicians when rDTAA is suspected. ${ }^{13}$

Our study has certain limitations related to the source of our data. First, we used an administrative database that is subject to coding errors, because data entered by coders are likely to be biased by the constraints of using only ICD-9-CM codes for data reporting. However, using a current clinical database that is more robust (eg, the Society of Thoracic Surgeons database) was not an option, because such databases have only recently begun to collect detailed information on TEVAR procedures. In addition, cardiologists and vascular surgeons who perform TEVAR do not participate in Society of Thoracic Surgeons data reporting, making the NIS the only data source that captures all TEVAR procedures across the nation.

Second, the NIS database does not capture data regarding readmissions, reinterventions, or long-term outcomes. For these reasons and others, clinical decisions should not be based solely on the outcomes reported in an administrative database but should be supported by clinical judgment and standards of care. Nonetheless, our study provides important insight into the impact of TEVAR and hospital characteristics on solid end points, such as mortality, length of stay, and disposition.

It is also worth mentioning that of the smaller hospitals represented in the study, only $22 \%$ (6/27) performed TEVAR for rDTAA; the rest performed OAR exclusively. Some of these hospitals may have performed only OAR because, in the cases treated there, patient anatomy precluded the use of TEVAR; however, it is probable that at least some smaller hospitals performed OAR exclusively because they did not have the necessary personnel and equipment to perform TEVAR. In addition, a smaller hospital that has the resources needed to acquire TEVAR capability may have more resources in general than other smaller hospitals and may therefore be able to provide a higher quality of care. This difference in hospital culture may have confounded some of our findings.

Also, rDTAA constitutes a minute subset of the thoracic aneurysms treated each year in the United States. As a result, the number of patients with rDTAA in our study was relatively small. Therefore, although adjusting for case volume, surgeon volume, and surgeon specialty would have been ideal, we could not have performed an adequately powered statistical analysis if we had attempted to make such adjustments.

In addition, the NIS database does not capture variables related to the severity of rupture, such as whether patients were in shock at the time of presentation or whether the rupture was contained. Lack of such information made us unable to better stratify the patients for risk-adjusted analysis. Also, the diagnosis of rupture is sometimes presumed when a pleural effusion is noted on imaging studies, but this finding can indicate a sympathetic effusion rather than a true rupture. This distinction could not be made in our patients.

The increased rate of respiratory and renal complications we found in the TEVAR group is another surprising finding that differs from published data. ${ }^{2}$ Although this finding may be partly due to the higher comorbidity index in the patients in the TEVAR group, it is also likely that patients in the TEVAR group were in a more advanced state of shock on admission. The administrative nature of the NIS prevented us from better characterizing this anomalous finding. Likewise, the exact nature of postoperative neurologic complications cannot be determined from the NIS data.

Whereas the feasibility of TEVAR is subject to the anatomy of the aortic arch and the limitations of current endovascular devices, almost any part of the aorta can be repaired in an open fashion. The extent of the paradigm shift we shall witness in the future will depend on improvements in thoracic endograft designs, lessons learned from population-based studies, experience from centers of excellence, and surgeons' ability to overcome learning curves. A clinical study that evaluates the factors precluding the use of urgent TEVAR for rDTAA would be compelling at this juncture and would shed more light on the anatomic factors that influence surgeon decision-making in these special circumstances.

\section{CONCLUSIONS}

Smaller hospitals were associated with a higher overall complication rate for any intervention for rDTAA. The complication rate was lower for TEVAR procedures. Our results suggest that smaller hospitals handle open repairs less well than larger hospitals, so it would be prudent to transfer patients with rDTAA to a large hospital when transfer is feasible. However, any patient with a life-threatening emergency is likely to be taken to the nearest hospital, which may be smaller or larger, depending on its location, local emergency medical services policy, and other factors. rDTAA is rare and unlikely to be suspected before the patient reaches the emergency department. Valuable time has probably already been lost by the time transport to a facility with expertise in thoracic aortic surgery is considered. Unfortunately, safe transfer may not be possible in every case, especially if the patient is in extremis, so smaller hospitals may be forced to handle such complex cases on occasion. If these hospitals possess endovascular facilities, then the use of TEVAR may be a better alternative, given the poorer outcomes typically seen with OAR in smaller hospitals. Thus, our findings suggest the importance of conducting a randomized trial to evaluate the feasibility of endovascular thoracic aortic intervention in smaller hospitals. Such a trial would help to determine whether endovascular suites in smaller hospitals are capable of handling rDTAA cases and delivering outcomes similar to those of larger centers. The trial could also reveal whether it would 
be beneficial to develop nationwide, uniform rapidassessment criteria for potential TEVAR intervention when rDTAA is suspected.

Although TEVAR should still be considered a highly advanced procedure that is best performed in a quaternary center, it should be acknowledged that we are on the verge of a paradigm shift. The steady increase in the adoption of TEVAR for rDTAA over a short span of 3 years, the better disposition rates of patients who have undergone this procedure, and the use of TEVAR for rDTAA even in smaller hospitals are noteworthy. The adoption of TEVAR for rDTAA is likely to follow similar trends observed in the adoption of endovascular approaches for treating ruptured abdominal aneurysms.

Stephen N. Palmer, PhD, ELS, contributed to editing the manuscript.

\section{References}

1. Makaroun MS, Dillavou ED, Kee ST, Sicard G, Chaikof E, Bavaria J, et al. Endovascular treatment of thoracic aortic aneurysms: results of the phase II multicenter trial of the GORE TAG thoracic endoprosthesis. $J$ Vasc Surg. 2005;41:1-9

2. Gopaldas RR, Huh J, Dao TK, LeMaire SA, Chu D, Bakaeen FG, et al. Superior nationwide outcomes of endovascular versus open repair for isolated descending thoracic aortic aneurysm in 11,669 patients. J Thorac Cardiovasc Surg. 2010; 140:1001-10.

3. Makaroun MS, Dillavou ED, Wheatley GH, Cambria RP. Five-year results of endovascular treatment with the Gore TAG device compared with open repair of thoracic aortic aneurysms. J Vasc Surg. 2008;47:912-8.

4. Bhamidipati CM, Lapar DJ, Mehta GS, Kern JA, Kron IL, Upchurch GR Jr, et al. Have thoracic endografting outcomes improved since US Food and Drug Administration approval? Ann Thorac Surg. 2011;91:1314-22.

5. Cooper DG, Walsh SR, Sadat U, Hayes PD, Boyle JR. Treating the thoracic aorta in Marfan syndrome: surgery or TEVAR? J Endovasc Ther. 2009;16:60-70.

6. Steuer J, Eriksson MO, Nyman R, Bjorck M, Wanhainen A. Early and long-term outcome after thoracic endovascular aortic repair (TEVAR) for acute complicated type B aortic dissection. Eur J Vasc Endovasc Surg. 2011;41:318-23.

7. Tang DG, Dake MD. TEVAR for acute uncomplicated aortic dissection: immediate repair versus medical therapy. Semin Vasc Surg. 2009;22:145-51.

8. Chemelli-Steingruber IE, Chemelli A, Strasak A, Hugl B, Hiemetzberger R, Czermak BV. Evaluation of volumetric measurements in patients with acute type B aortic dissection-thoracic endovascular aortic repair (TEVAR) vs conservative. J Vasc Surg. 2009;49:20-8.

9. Hughes GC, Lee SM, Daneshmand MA, Bhattacharya SD, Williams JB, Tucker SW Jr, et al. Endovascular repair of descending thoracic aneurysms: results with "on-label" application in the post Food and Drug Administration approval era. Ann Thorac Surg. 2010;90:83-9.

10. Patel HJ, Williams DM, Upchurch GR Jr, Dasika NL, Deeb GM. A comparative analysis of open and endovascular repair for the ruptured descending thoracic aorta. J Vasc Surg. 2009;50:1265-70.

11. Starnes BW, Quiroga E, Hutter C, Tran NT, Hatsukami T, Meissner M, et al. Management of ruptured abdominal aortic aneurysm in the endovascular era. J Vasc Surg. 2010;51:9-18.

12. Jonker FH, Verhagen HJ, Lin PH, Heijmen RH, Trimarchi S, Lee WA, et al. Outcomes of endovascular repair of ruptured descending thoracic aortic aneurysms. Circulation. 2010;121:2718-23.

13. Coselli JS, Gopaldas RR. Ruptured thoracic aneurysms: to stent or not to stent? Circulation. 2010;121:2705-7.

14. Coady MA, Ikonomidis JS, Cheung AT, Matsumoto AH, Dake MD, Chaikof EL, et al. Surgical management of descending thoracic aortic disease: open and endovascular approaches. A scientific statement from the American Heart Association. Circulation. 2010;121:2780-804.

15. Coselli JS, LeMaire SA, Conklin LD, Adams GJ. Left heart bypass during descending thoracic aortic aneurysm repair does not reduce the incidence of paraplegia. Ann Thorac Surg. 2004;77:1298-303.
16. Healthcare Cost Utilization Project, Nationwide Inpatient Sample (NIS). Rockville, MD: Agency for Healthcare Research and Quality; 2001.

17. Healthcare Cost and Utilization Project website: Nationwide Inpatient Sample section. Available at: http://www.hcup-us.ahrq.gov/db/nation/nis/nisrelatedreports.jsp. Accessed January 5, 2011.

18. International Classification of Diseases, Ninth Revision, Clinical Modification. 5th ed. Washington, DC: US Department of Health and Human Services, Public Health Service; 1988.

19. Orandi BJ, Dimick JB, Deeb GM, Patel HJ, Upchurch GR Jr. A population-based analysis of endovascular versus open thoracic aortic aneurysm repair. $J$ Vasc Surg. 2009;49:1112-6.

20. Deyo RA, Cherkin DC, Ciol MA. Adapting a clinical comorbidity index for use with ICD-9-CM administrative databases. J Clin Epidemiol. 1992;45:613-9.

21. Deyo RA, Taylor VM, Diehr P, Conrad D, Cherkin DC, Ciol M, et al. Analysis of automated administrative and survey databases to study patterns and outcomes of care. Spine. 1994;19:2083S-91S.

22. Gopaldas RR, Bakaeen FG, Dao TK, Walsh GL, Swisher SG, Chu D. Video-assisted thoracoscopic versus open thoracotomy lobectomy in a cohort of 13,619 patients. Ann Thorac Surg. 2010;89:1563-70.

23. Gopaldas RR, Chu D, Dao TK, Huh J, LeMaire SA, Coselli JS, et al. Predictors of surgical mortality and discharge status after coronary artery bypass grafting in patients 80 years and older. Am J Surg. 2009;198:633-8.

24. Gopaldas RR, Chu D, Dao TK, Huh J, LeMaire SA, Coselli JS, et al. Impact of ACGME work-hour restrictions on the outcomes of coronary artery bypass grafting in a cohort of 600,000 patients. J Surg Res. 2010;163:201-9.

25. Gopaldas RR, Chu D, Dao TK, Huh J, LeMaire SA, Lin P, et al. Staged versus synchronous carotid endarterectomy and coronary artery bypass grafting: analysis of 10-year nationwide outcomes. Ann Thorac Surg. 2011;91:1323-9.

\section{Discussion}

Dr Thomas Gleason (Pittsburgh, Pa). I have no disclosures. Dr Gopaldas, this was an excellent presentation and a well-written article. I have a few questions.

First, your primary inclusion code was for ruptured thoracic aneurysm, which includes, of course, ascending, arch, and descending aortic aneurysms, each of which could significantly affect treatment strategy. How did you ensure that only treatment of descending thoracic aneurysms was included in your analysis? If inclusion was based on trapping those in whom there was also use of cardiopulmonary bypass, this would also not guarantee exclusion of ascending or arch aneurysms.

Dr Gopaldas. The NIS database captures procedure codes, and we did use the code that identifies cardiopulmonary bypass with the use of an oxygenator. This is different from left heart bypass. So we did exclude all patients who underwent cardiopulmonary bypass. That ensured all patients with ascending and arch aneurysms were excluded. The one downside to that, however, is a subset of patients who underwent isolated descending repair under circulatory arrest were probably excluded.

Now, if there was a way to include that particular subset, it would have made our analysis stronger, but these are probably a sicker subgroup of patients, and if they were included in the model, it probably would have made TEVAR look even better.

Dr Gleason. How about cost? Were you able to establish any estimates based on the NIS data used as to differences in hospital cost per patient per group?

Dr Gopaldas. We actually did a cost analysis. I did not include the slide. There was no statistically significant difference in cost between TEVAR and open procedures, and even with the risk-adjusted model we did not find a difference. The approximate cost was $\$ 20,000$ for TEVAR and $\$ 19,000$ for open repair, a $\$ 1000$ difference. But, again, this was not statistically significant. 
Dr Gleason. In the article, I note that respiratory complications were actually significantly higher in the TEVAR group compared with the open group, but despite this fact, length of stay and overall mortality were lower in that group. Do you have any insight into this apparent discrepancy?

Dr Gopoldas. That is a good question, and I was surprised when we looked through the data. When we ran the analysis for the first time, we were surprised partly because our earlier results and studies on elective cases were exactly the opposite, where the respiratory complications were much lower. But the thing to look into and pay attention to was that respiratory complications also include pleural effusions or hemothorax and interventions for that. In an elective case you probably don't have to deal with much of those, but when these patients come in with a rupture or potential rupture, a lot of them have a pleural effusion. When they undergo an open repair, they automatically have chest tubes placed, and so pleural effusion is not documented separately. But then when they undergo TEVAR and still have a persistent pleural effusion, it was documented for Diagnosis-Related Group billing purposes. They probably had a thoracentesis and chest tube, and as a result of that, the percentage of respiratory complications was higher. When we eliminated pleural effusion, the respiratory complications actually were lower in the TEVAR subgroup.

Dr Gleason. We are not provided with the relative difference in the number of open and TEVAR in the smaller and larger hospitals in the article or presentation. I am curious whether there was a difference in the percentage of approach by size of hospital. Finally, if this crude but large data set and analysis can be validated, what conclusion should be drawn with respect to the large centers in particular? It seems this may represent justification for more rather than less use of open techniques in experienced centers if the mortality and complication rates are shown to be equivalent, as you have. Given that Dr Coselli's group has one of the largest practices in the world for this disease set, how do you look at this data set and respond at your own institution? How should this new information change a large center's practice?

Dr Gopaldas. Those are valid questions, and the point is well taken. In cases performed at larger hospitals, $40 \%$ of them were TEVAR, and in smaller hospitals it was approximately $25 \%$, and there was a statistically significant difference between them.

Now, to answer your question about how large centers should look at this: I think the focus here was that the significant difference we noticed was in smaller centers where they had a tactical advantage in performing TEVAR compared with open repair. Although we did not show a difference in the outcomes in large hospitals between TEVAR and open repair, the one thing we have to look at is the disposition of these patients. There was a 3 times greater chance that these patients actually went home after a TEVAR when they were in a large hospital. Although there is no difference in mortality, if you are looking at an elderly subgroup of patients who are discharged and end up in a nursing home after an open repair, you are dealing with a lot of problems down the road, such as bedsores, pulmonary problems, and probably being debilitated and not going home. So I think TEVAR still has an advantage in that particular respect.

I did my cardiothoracic residency at Baylor, spent 3 years with Dr Coselli, and was honored for having spent time there. The way things are handled there is streamlined. There is a protocol in place in the emergency department. So if anybody comes to the emergency department and a question of an aortic issue is raised, they are streamlined for a computed tomography angiogram rather immediately. If the anatomy is feasible, we always prefer to use TEVAR as our first option in these circumstances, because it is quicker to do a femoral artery cutdown and tackle this as long as the anatomy is feasible and allows that.

Now, I have been at the University of Missouri for less than 1 year, and we don't have a hybrid suite; we are building one now. I have been involved in approximately 12 cases, 2 of them were ruptured TEVARs and the others were aortic transections. The one thing I can tell from my limited experience is that it is a lot easier in the middle of the night to work with an endovascular team, because they are in the hospital almost every day dealing with a cardiac catheter laboratory emergency or peripheral vascular emergency. On the other hand, if I have to mobilize the open heart team to come in, it takes at least 1 to 1.5 hours. I would rather use that time to try an endovascular approach while I get the open heart team in.

So with the availability of the technology and these data, TEVAR should be the ideal approach as long as you have a suitable anatomy and the proper imaging studies.

Dr Gorav Ailawadi (Charlottesville, Va). Using the NIS to compare 2 different techniques is not ideal because we really don't have any information about the anatomy. There is significant selection bias. You cannot use NIS to compare 2 different procedures and conclude that one is better than another because of these significant limitations with the database. But my question relates to the methodology of your inclusion/exclusion related to the patients that you called "hybrid." You can use ICD-9-CM codes to tell us if they had TEVAR or open. What did you do if they had both? Did you exclude them because you said they were hybrid, or what if they had a TEVAR and then underwent an open procedure because of a complication or vice versa during the same hospitalization? How can you distinguish this? There really isn't a great way to define which day during the hospitalization a particular procedure occurred. If you exclude these patients, then you ignore complications that may have occurred with either approach.

Dr Gopaldas. We excluded all patients who had both procedures done in the same hospital admission. The NIS will allow you to identify the time difference between the 2 procedures, because it does have a procedure day code. The problem, however, is that we can't tell if the patient had an open approach first and subsequently had a problem such as a pseudoaneurysm or an anastomotic leak and needed to have a TEVAR done, or if they had a TEVAR done first and then it had to be converted to an open procedure later. Because we couldn't differentiate these 2 subcategories, we decided to exclude all patients who had both procedures done in the same hospital admission. 
APPENDIX 1. ICD-9-CM codes used for inclusion/exclusion of study group patients

Codes used for inclusion

441.1 Thoracic aneurysm, ruptured

39.73 Procedure code: Endovascular implantation of graft in thoracic aorta

38.45 Procedure code: Resection of vessel with replacement; thoracic aorta

Diagnosis codes used for exclusion

441.2

441.3

441.4

441.5

441.6

441.7

441.9

441.00

441.01

441.02

441.03

446.0

446.1

446.2

446.3

446.4

446.5

446.6

446.7

758.6

759.82

Thoracic aneurysm without mention of rupture

Abdominal aneurysm, ruptured

Abdominal aneurysm without mention of rupture

Aortic aneurysm of unspecified site, ruptured

Thoracoabdominal aneurysm, ruptured

Thoracoabdominal aneurysm without mention of rupture

Aortic aneurysm of unspecified site without mention of rupture

Aortic dissection, unspecified site

Aortic dissection, thoracic

Aortic dissection, abdominal

Aortic dissection, thoracoabdominal

Polyarteritis nodosa

Kawasaki disease

Hypersensitivity angiitis

Lethal midline granuloma

Wegener's granulomatosis

Giant cell arteritis

Thrombotic microangiopathy

Takayasu disease; aortic arch arteritis

Turner syndrome; gonadal dysgenesis

Marfan syndrome

Procedure codes used for exclusion

$39.61 \quad$ Cardiopulmonary bypass auxiliary to open procedure

$39.73+38.45$ Indicates conversion during or reintervention after a thoracic aneurysm repair

39.71

Endovascular implantation of graft in abdominal aorta

38.44

Resection of vessel with replacement; abdominal aorta
APPENDIX 2. ICD-9-CM codes used to identify procedure-related complications

\begin{tabular}{ll}
\hline 423.0 & Hemopericardium \\
997.1 & Cardiac arrest or cardiorespiratory failure \\
996.0 & Mechanical complication due to an implant/graft \\
996.74 & Thrombosis, fibrosis, hemorrhage, or embolism due to \\
& $\quad$ a vascular graft \\
998.31 & Disruption of surgical wound \\
997.71 & Vascular complications of mesenteric artery \\
998.0 & Postoperative shock \\
998.11 & Hemorrhage \\
998.12 & Hematoma \\
998.13 & Seroma \\
E878.2 & Abnormal patient reaction/complication resulting directly \\
& from a surgical procedure involving placement of \\
& a prosthetic vascular graft \\
\hline
\end{tabular}

